\title{
Le radon, un traceur atmosphérique
}

\section{Grâce à sa radioactivité d'origine naturelle, le radon produit dans la croûte terrestre apporte déjà des informations essentielles sur le transport atmosphérique.}

Désormais, il permet également de suivre les flux de gaz à effet de serre dans l'atmosphère. Les chercheurs espèrent que ce gaz noble, en tant que traceur de masses d'air, permettra également une meilleure quantification des rejets et des flux de gaz à effet de serre tels que le $\mathrm{CO}_{2}$.

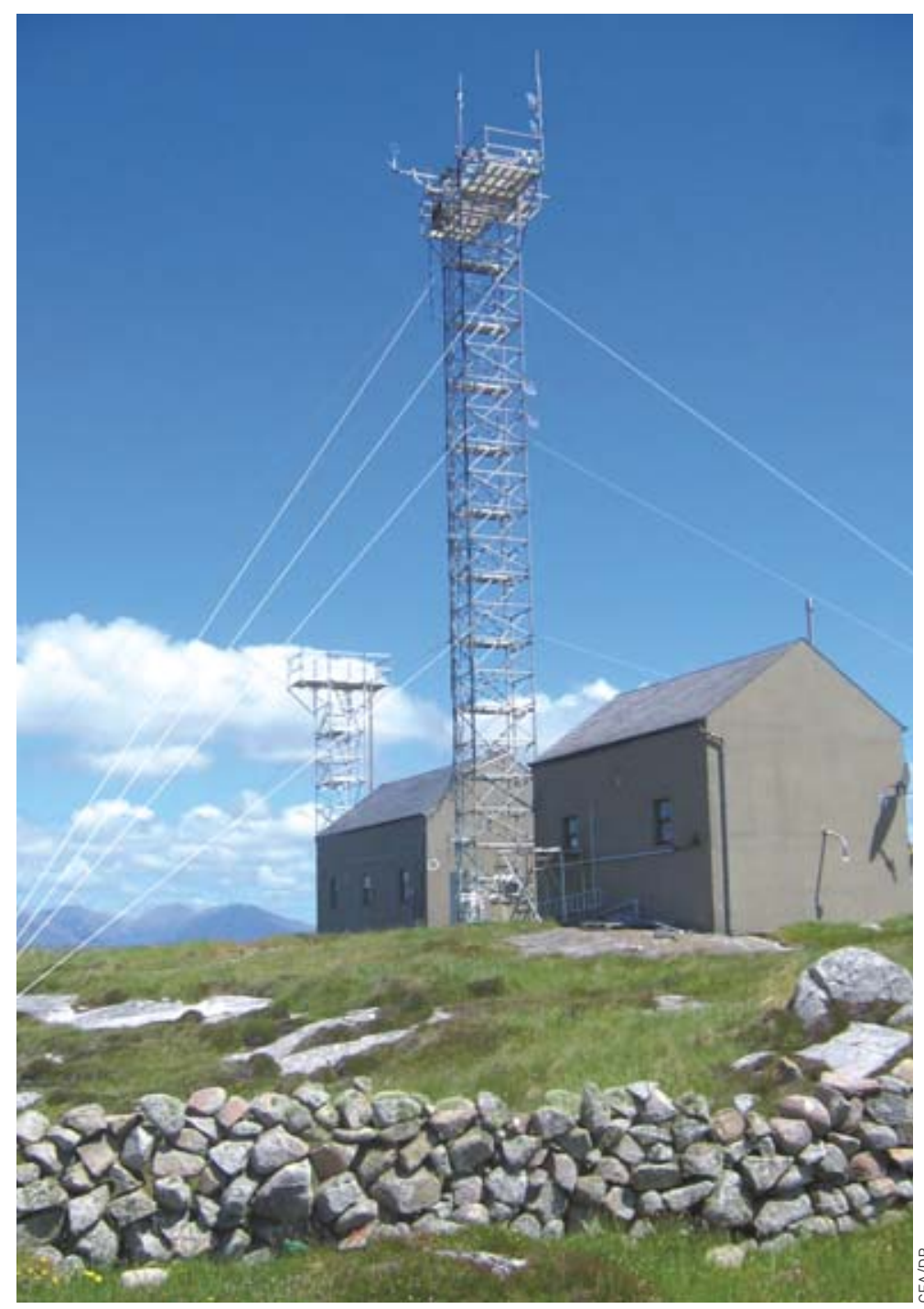

Observatoire de Mace Head (Irlande).

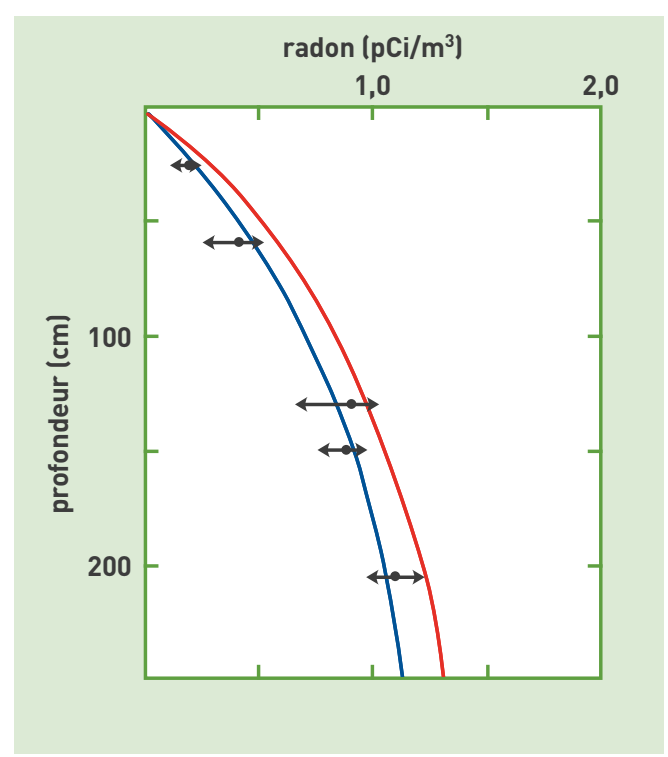

Figure 1.

Variation typique des concentrations de ${ }^{222} \mathrm{Rn}$ en fonction de la profondeur du sol. L'axe des abscisses indique la concentration de ${ }^{222} \mathrm{Rn}\left(\mathrm{pCi} / \mathrm{cm}^{3}\right)$ mesuré dans le sol $(1 \mathrm{pCi}=0,037 \mathrm{~Bq})$ tandis que l'axe des ordonnées indique la profondeur du sol $(\mathrm{cm})$. Notez la décroissance des concentrations à proximité de la surface.

sance des radionucléides sont alors sensiblement les mêmes et ils correspondent à une production de 0,7 à 7 atomes de radon (radon 222) par minute et par gramme de matériau. Une infime partie de ce radon produit par les roches parvient à s'échapper dans l'atmosphère, et quand il n'y parvient pas, l'atome de radon en contact avec la surface terrestre se transforme en suivant la chaîne de l'uranium. Des mesures, réalisées à la surface de la Terre et sur de larges étendues, ont montré que le flux de radon s'établit en moyenne à 1 atome $/ \mathrm{cm}^{2} / \mathrm{s}$ (figure 1 ). Tandis que les chercheurs du CEA, emmenés par Jacques Labeyrie et Gérard Lambert, procédaient à des mesures de flux de radon provenant des sols puis de ses concentrations présentes dans l'atmosphère, l'équipe de Wally Broecker du Lamont Doherty Earth Laboratory, de l'Université de Columbia (New York), relevait des quantités encore plus petites de radon dansl'eau de mer. Des calculs, prenant en compte les échanges gazeux entre océan et atmosphère, ont également montré que le flux de radon provenant de l'océan s'avérait 100 fois inférieur à celui résultant des surfaces continentales. Cette 1 à 10 parties par million), l'uranium se trouve en équilibre radioactif avec tous ses descendants, jusqu'au radium. Les taux de formation et de décrois- 
différence, observée entre les flux océaniques et terrestres, a permis aux chercheurs d'éclaircir plusieurs aspects de la dynamique et du transport atmosphérique jusqu'alors incompris. Ainsi, sans les études menées sur le radon, de précieux renseignements n'auraient jamais pu être collectés. Les découvertes réalisées depuis ces trois dernières décennies ont donc permis de résoudre une série d'interrogations.

\section{Masses d'air dans les océans : temps de transit et périodes d'échanges actifs}

Concernant les océans Atlantique, Pacifique, Indien et circumpolaire Antartique, il faut savoir qu'en raison de la répartition des masses terrestres à la surface du globe mais aussi des flux de radon se situant autour d'un atome $/ \mathrm{cm}^{2} / \mathrm{s}$ au-dessus des terres émergées, les concentrations de radon y varient, en moyenne, entre 35 et $70 \mathrm{mBq} / \mathrm{m}^{3}$ (figure 2). Ensuite, il faut également prendre en compte la période radioactive du radon, laquelle s'établit à 3,8 jours. L'ensemble de ces données permettent de comprendre que la concentration d'une masse d'air, en quittant un continent avec une concentration de $50 \mathrm{mBq} / \mathrm{m}^{3}$, puis en transitant 10 jours au-dessus des océans sans jamais rencontrer de continent, décroît à $8,1 \mathrm{mBq} / \mathrm{m}^{3}$. Ces résultats illustrent comment, en l'absence de toute dilution, la concentration en radon de cet air permet de dater sa dernière rencontre avec un continent. Cette propriété essentielle du radon a permis de démontrer que certaines masses d'air traversent l'océan Pacifique, en moins de cinq jours, c'est-à-dire trois fois plus rapidement que n'importe quel autre moyen de navigation maritime. Pour expliquer ces temps de transit record, les chercheurs ont réalisé des mesures à partir d'avions spécialement équipés pour collecter l'air en hautes altitudes. Leurs résultats établissent, qu'au printemps et en été, l'air situé à la surface du continent asiatique se trouve d'abord très rapidement transporté en altitude, puis projeté, par jet d'altitude (vers $10 \mathrm{~km}$ ), au travers du Pacifique nord. Repris par des courants

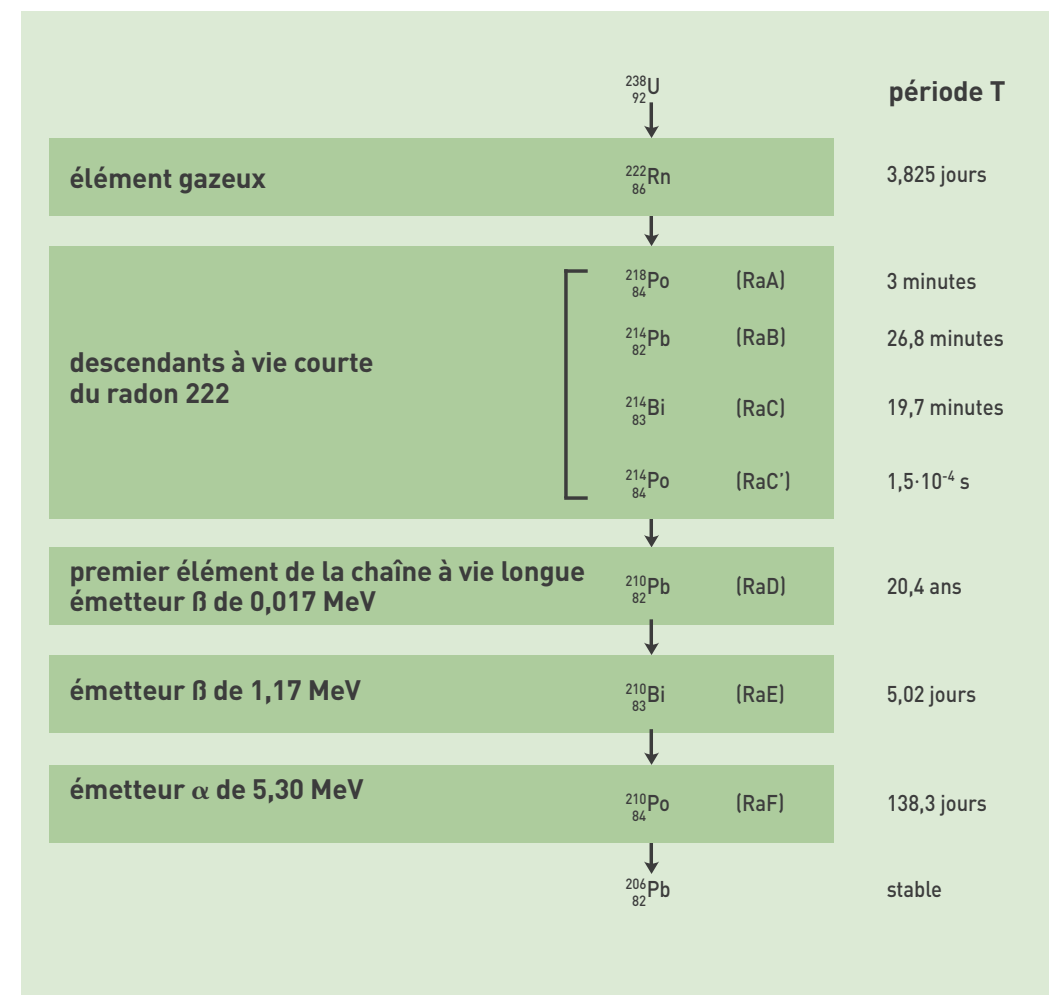

descendants, cet air atteindra ensuite les couches basses atmosphériques situées au-dessus du continent Nord-américain. Ainsi, grâce à l'introduction des mesures et à la modélisation du radon dans des modèles tridimensionnels de l'atmosphère, les chercheurs ont pu mettre en évidence le mécanisme majeur des échanges de polluants inter-continents.

\section{Temps d'échange entre les basses et les plus hautes couches de l'atmosphère}

Plusieurs profils verticaux de concentrations de radon, mesurés au-dessus des continents, témoignent que le nombre d'atomes décroît d'un facteur 10 à 100 en moyenne, au fur et à mesure que l'on s'élève
Tableau.

Chaîne des descendants du radon 222 .

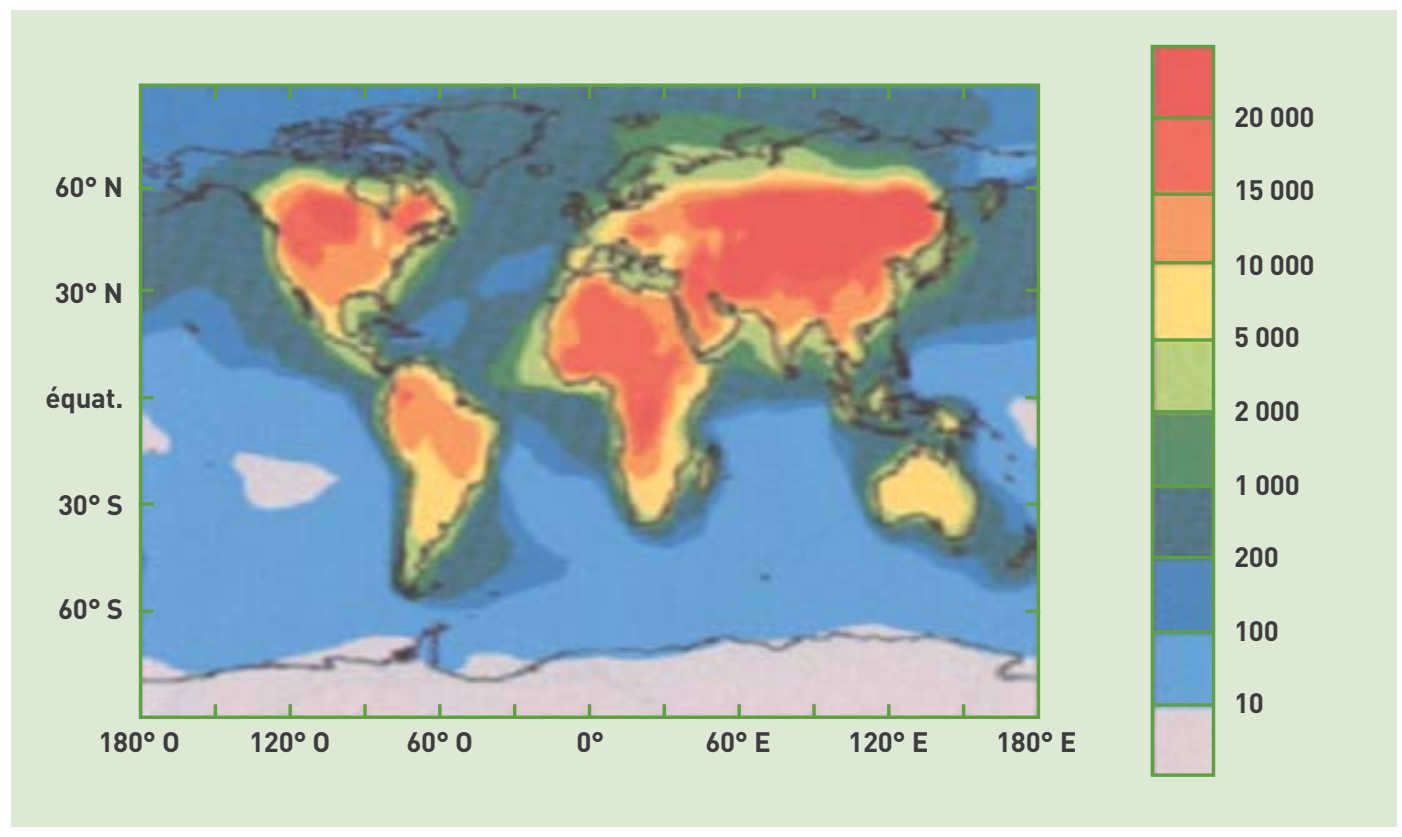

Figure 2.

Concentration de radon dans l'air à la surface de l'atmosphère $\left(\mathrm{mBq} \cdot \mathrm{m}^{-3}\right)$. Il existe de fort gradients de concentration entre les continents et les océans. 


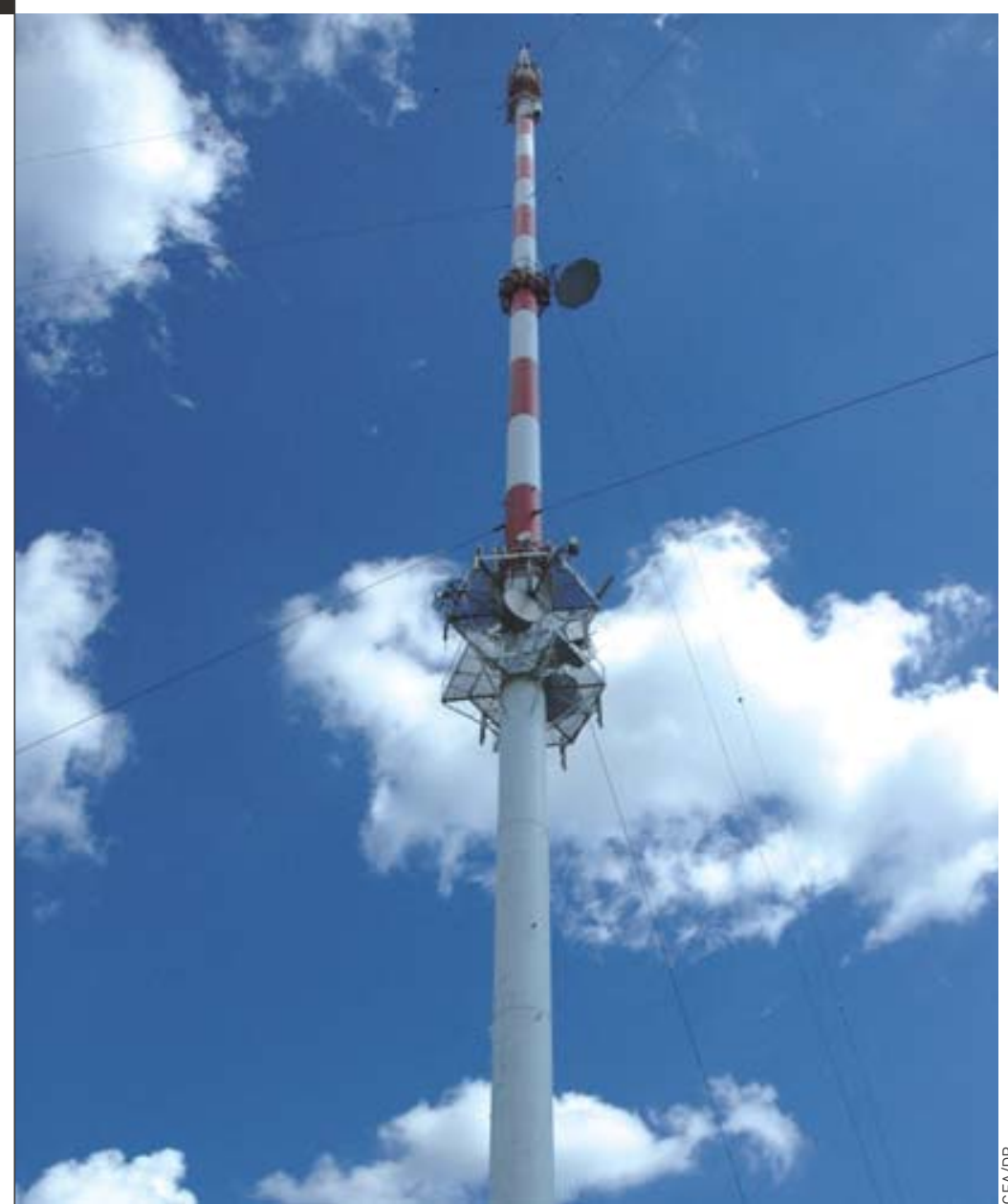

La tour du Traînou, dans la forêt d'Orléans, à partir de laquelle les équipes du LSCE mesurent le $\mathrm{CO}_{2}$. Son sommet s'élève à 180 mètres.

au-dessus de la surface de la Terre, et cela jusqu'à une altitude de 12 kilomètres. Le regroupement de ces profits verticaux ainsi que les moyennes établies pour les deux saisons d'été et d'hiver ont laissé apparaître une image contrastée : de 0 à 12 kilomètres, la décroissance en radon s'avère de l'ordre de 100, en hiver, contre seulement 10 en été. C'est grâce à la modélisation des processus atmosphériques qu'il a été permis de comprendre cette différence saisonnière puis de démontrer sa liaison étroite avec le phénomène de convection se produisant au-dessus des continents. Ainsi, en été, au moment où s'échangent d'importantes quantités de chaleur sensible avec l'atmosphère, l'air passe rapidement de la surface vers les couches les plus hautes : par exemple, une parcelle d'air ayant été en contact récent avec la surface peut se trouver échangée dans des courants convectifs ascendants, et cela jusqu'à 10 kilomètres d'altitude. En raison de ce contact rapide avec la surface, l'air d'altitude s'enrichira plus fortement en radon l'été que l'hiver. En altitude la différence de concentration de radon observée entre ces deux saisons, découle donc de phénomènes convectifs.

\section{“Orages radoniques" sur l'océan Indien}

C'est en mesurant le radon de manière routinière dans les îles australes (Crozet, Kerguelen, Amsterdam) et à Dumont d'Urville (côte Antarctique) que Georges Polian, ingénieur de recherche au CNRS, observa des épisodes, d'une dizaine d'heures environ, pendant lesquelles les concentrations de radon devenaient tout à fait inhabituelles puisqu'elles dépassaient de 10 à 30 fois le bruit de fond régulier mesuré tout au long de l'année. Ce chercheur donna à ces épisodes le nom singulier d' "orages radoniques". Ensuite, il avança que le radon mesuré ne provenait pas du sol des îles Australes en utilisant l'isotope 220 de ce gaz.

Restait alors à découvrir l'origine de ces fortes augmentations observées dans les phénomènes de transport à longue distance. Ce sont deux équipes des universités d'Harvard et d'Hambourg qui expliqueront, indépendamment, la cause de ces "orages radoniques” en recourant à des modèles de transport atmosphérique. C'est ainsi que ces chercheurs ont pu établir que la formation de ce phénomène s'effectuait à partir de masses d'air ayant été en contact récent avec le continent africain avant de se trouver rapidement transportées vers les îles de Crozet, Kerguelen et Amsterdam par advection. À ce moment-là, l'air se trouve expulsé du continent en raison du passage d'une basse pression localisée au sud de l'Afrique du Sud, créée au voisinage de la haute pression du Mascarène située près de Madagascar. S'ensuit un très fort appel d'air opérant vers le large avant de transiter del'Afrique aux îles subantarctiques en quelques jours.

\section{Variation des concentrations de radon entre hiver et été}

En général, les mesures de radon effectuées au-dessus des sites continentaux montrent des concentrations nettement plus élevées en hiver qu'en été. A priori, cette différence ne proviendrait pas des variations de flux saisonnières mais plutôt de la ventilation opérant entre les basses couches atmosphériques (inférieures à $2 \mathrm{~km}$ ) et les couches plus hautes (entre 2 et $12 \mathrm{~km}$ ). En effet, l'hiver, les échanges entre les niveaux bas et la troposphère libre s'avèrent peu importants, tandis que l'été, ces échanges deviennent beaucoup plus intenses. En conséquence, le radon tend alors à se confiner dans les plus basses couches atmosphériques faisant que les concentrations proches du sol s'élèvent. En revanche, l'été, les échanges entre la surface et la troposphère amènent, des hautes altitudes à la surface, un air pauvre en radon qui provoque une dilution des concentrations et explique l'observation de concentrations plus basses.

\section{Le radon pour mieux quantifier les flux de $\mathrm{CO}_{2}$ provenant des sols}

La capacité des chercheurs à pouvoir mesurer les flux de gaz à effet de serre avec précision figure comme l'un des grands enjeux du futur pour mieux appréhender les changements climatiques. Mais, bien cerner les flux émis par l'homme suppose préalablement de quantifier les flux naturels de gaz tels que le $\mathrm{CO}_{2}$ et le méthane. Parmi les techniques existantes, celle de l'Eddy-corrélation consiste à mesurer très précisément les concentrations des gaz à deux niveaux d'altitude différents : les différences enregistrées entre ces deux niveaux permettent ensuite de déduire un flux instantané. Pour illustrer simplement la manière de déduire ce flux à partir de concentrations, il suffit de supposer que $h$ soit la hauteur de 


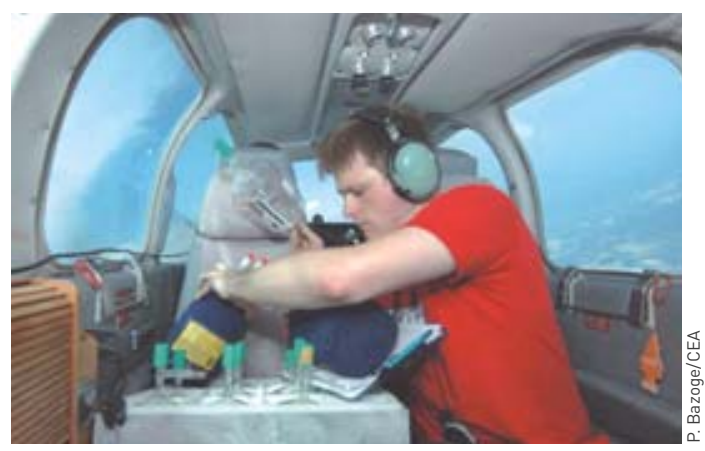

Prélèvements d'air au-dessus de la forêt d'Orléans.

la couche limite atmosphérique, découplée des couches atmosphériques supérieures et située près de la surface dans laquelle ces gaz sont émis (typiquement entre 0,5 et $2 \mathrm{~km}$ ); la variation de la concentration au temps $t, C(t)$, d'un traceur atmosphérique bien mélangé dans cette couche atmosphérique dépend alors seulement de la hauteur, $h$, et peut s'exprimer comme :

$\Delta C(t)=\frac{F}{h} t$,

où $\Delta C(t)$ représente la variation de concentration observée entre l'instant 0 et l'instant $t$. $F$, le flux du gaz étudié, est supposé constant sur l'intervalle de temps mesuré. La même hypothèse vaut pour la hauteur $h$ de la couche limite atmosphérique. Enfin, le flux d'échanges entre les couches atmosphériques basses (jusqu'à 2,5 km) et plus hautes (troposphère libre, entre 2,5 et $10 \mathrm{~km}$ environ) est supposé nul. Malheureusement, le flux mesuré par cette technique n'est calculé que pour une superficie faible autour du point de la mesure (typiquement $1 \mathrm{~km}^{2}$ pour des conditions atmosphériques instables). Le flux à quelques kilomètres du lieu de mesure peut être très différent. Assez tôt, l'idée a donc émergé que des mesures précises de concentrations pourraient permettre de quantifier les flux de gaz tels que le $\mathrm{CO}_{2}$, le $\mathrm{CH}_{4}$ et le $\mathrm{N}_{2} \mathrm{O}$ grâce à une assez bonne connaissance du flux $\mathrm{de}^{222} \mathrm{Rn}$ sur de larges échelles (del'ordre de $100 \mathrm{~km}^{2}$ ). Supposons donc que le flux de radon au-dessus de la région considérée soit connu avec une bonne précision (supérieure à $30 \%$, par exemple), on peut alors exprimer le rapport des fluctuations des concentrations entre les deux gaz, le $\mathrm{CO}_{2}$ et le radon, comme :

$F_{\mathrm{CO}_{2}}=F_{222} \mathrm{Rn} \frac{\Delta C_{\mathrm{CO}_{2}}}{\Delta C_{222} \mathrm{Rn}}$

Une correction permet de prendre en compte le fait que les océans n'émettent pas de radon. L'exactitude de cette méthode suppose la réunion de plusieurs conditions : - un flux de radon presque constant au-dessus de toute la surface considérée ;

- des sources de radon et de $\mathrm{CO}_{2}$ variant spatialement de manière identique ;

- la connaissance du temps passé par le radon audessus des océans ;

- des propriétés identiques pour les deux gaz vis-àvis de leur destruction ou de leur production dans l'atmosphère.

Bien que les deuxième et quatrième conditions ne s'avèrent pas complètement satisfaites, plusieurs équipes du CEA et d'autres instituts ont pu tirer profit de cette méthode pour mesurer les gaz à effet de serre.

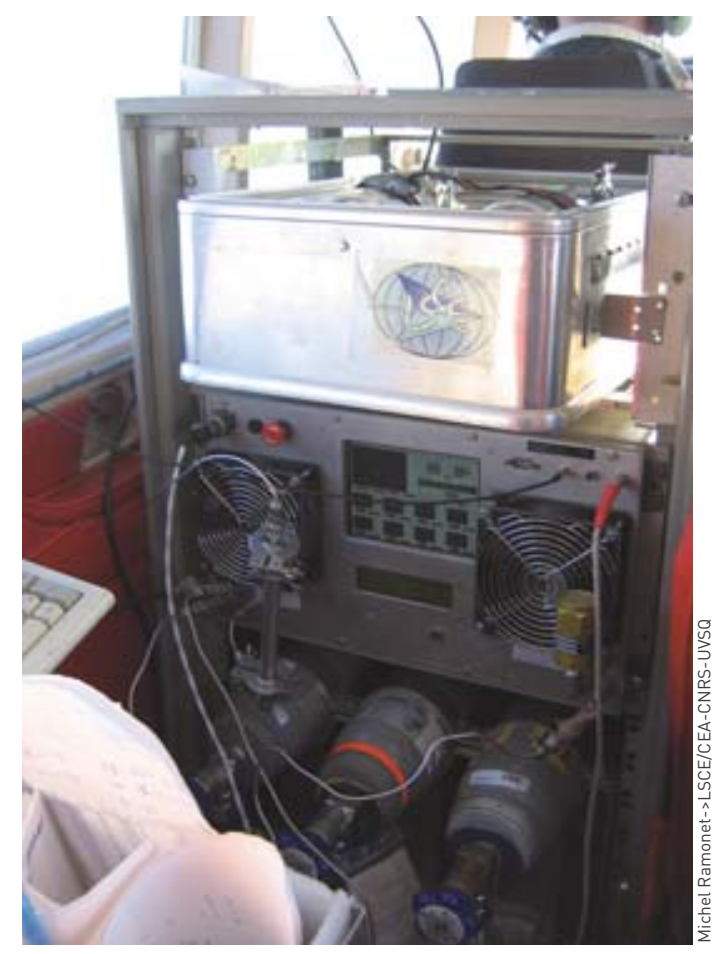

Système de mesure aéroporté du $\mathrm{CO}_{2}$ atmosphérique. En haut, la mallette de prélèvement de flacons; au centre, l'instrument de mesure du $\mathrm{CO}_{2}$ en continu, baptisé Condor ; en bas, les bouteilles d'air comprimé utilisées comme référence par l'instrument.

En conclusion, le radon apporte des informations essentielles à la compréhension du transport atmosphérique. Gaz noble, il s'utilise aussi bien pour l'étude des processus dynamiques de l'atmosphère que pour la compréhension de transports à longue distance. Plus récemment, une nouvelle voie très prometteuse s'est ouverte qui permettra, sans doute, de quantifier les rejets de gaz à effet de serre et de mieux mettre en œuvre les actions susceptibles d'endiguer le changement climatique.

$>$ Yves Balkanski

Laboratoire des sciences du climat et de l'environnement/Institut Pierre-Simon Laplace Unité mixte de recherche CEA-CNRS-UVSQ

Direction des sciences de la matière CEA Centre de Saclay (Orme des Merisiers)

\section{POUR EN SAVOIR PLUS}

Y. BALKANSKI, D. JACOB, R. ARIMOTO and M. KRITZ, "Distribution of Rn-222 Over the North Pacific: Implications for Continental Influences", J. Atmosph. Chem,

14, pp. 353-374, 1992.

S. Biraud, P. CiaIS, M. Ramonet, P. Simmonds, V. Kazan, P. Monfray, S. O'Doherty, T-G. SPAIN and S-G JENNINGS, "European greenhouse gas emissions estimated from continuous atmospheric measurements and radon 222 at Mace Head, Ireland", J. Geophys. Res., 105(D1), 1351-1366, 2000.

A. GAudry, G. Polian, B. Ardouin, G. LAMBERT, “Radon-calibrated emissions of $\mathrm{CO}_{2}$ from South Africa", Tellus 42B, 9-19, 1990.

M. Heimann, P. Monfray, G. Polian, “Modeling the long-range transport of $222 \mathrm{Rn}$ to subantarctic and Antarctic areas", Tellus 42B, 83-99, 1990.

W. Zahorowski, S.-D. Chambers and A. Henderson-Sellers, “Ground based radon-222 observations and their application to atmospheric studies", J. Environ. Radioactivity, 76, 3-33, 2004. 